\title{
MS049.P01
}

\section{Extended range USAXS/SAXS/WAXS for advanced alloy development}

Jan Ilavsky ${ }^{1}$, Fan Zhang ${ }^{2}$, Lyle Levine ${ }^{2}$, Andrew Allen ${ }^{2}$, Govindarajan Muralidharan ${ }^{3}$, Ross N. Andrews ${ }^{1}$

${ }^{1}$ Advanced Photon Source, ANL, Argonne, United States, ${ }^{2}$ National Institute of Standard and Technology, Gaithersburg, United States, ${ }^{3}$ Oak Ridge National Laboratory, Oak Ridge, United States

E-mail: ilavsky@aps.anl.gov

Development of new high-performance materials, e.g., new aluminum, nickel, or steel alloys, is critical for advances in energy production and utilization (and many other areas). These materials often exhibit complex microstructures spanning multiple length scales that control their performance. In this context, it is important to simultaneously characterize, ideally in situ or in operando, various facets of the microstructure - for example precipitate shape and size, together with their phase and chemical composition. Advanced Photon Source (APS), together with NIST, has developed and optimized a combined UltraSmall, Small, \& Wide Angle X-ray Scattering (USAXS/SAXS/WAXS) facility, [1]. Data spanning over 5 decades in microstructural size can be collected sequentially in 3 to 5 minutes, from the same volume during one in-situ experiment. We present case studies of $\mathrm{Al}-\mathrm{Cu}-\mathrm{Mg}$ alloys and $\mathrm{Ni}$-based superalloys studied at different aging conditions.

The precipitate structure and precipitation kinetics in the Al-Cu-Mg alloy, AA2024, aged at $190{ }^{\circ} \mathrm{C}, 208{ }^{\circ} \mathrm{C}$, and $226{ }^{\circ} \mathrm{C}$ have been studied using ex situ TEM and in situ combined USAXS/SAXS/WAXS across a length scale from sub-Angstrom to several micrometers, see Figure. TEM provided information concerning the nature, morphology, and size of the precipitates, while USAXS/SAXS/WAXS provided qualitative and quantitative information concerning the time-dependent size and volume fraction evolution of the precipitates at different stages of the precipitation sequence. Using a three-parameter scattering model constructed on the basis of the TEM results, we established the temperature-dependent kinetics for the clusterdissolution and S-phase formation processes. These two processes, while occurring simultaneously, have different kinetic rates, with the cluster-dissolution rate approximately double the S-phase formation rate. We identified activation energies and have shown the absence of an intermediate GPB2/S" phase in the samples under the experimental ageing conditions [2].

High performance Ni-based alloys for exhaust valve applications in future, higher efficiency, automotive engines need to be cost efficient while operate above $850^{\circ} \mathrm{C}$. We present results from simulated in-service conditions study of $\mathrm{Y}^{\prime}$ evolution.

Results from both studies document, how the extended-range USAXS/SAXS/WAXS facility simplifies and speeds up new advanced materials characterization and development.

Acknowledgement: This research used resources of the Advanced Photon Source, a U.S. Department of Energy (DOE) Office of Science User Facility operated for the DOE Office of Science by Argonne National Laboratory under Contract No. DEAC02-06CH11357.

[1] Ilavsky, J., Jemian, P.R., Allen, A.J., Zhang, F., Levine, L.E., and Long, G.G. (2009). Journal of Applied Crystallography, 42, 469-479.

[2] Zhang, F., Levine, L. E. , Allen, A. J., Campbell, C. E., Creuziger, A. A., Kazantseva, N. and Ilavsky, J. (2016). Acta Materialia 111, 385-398.

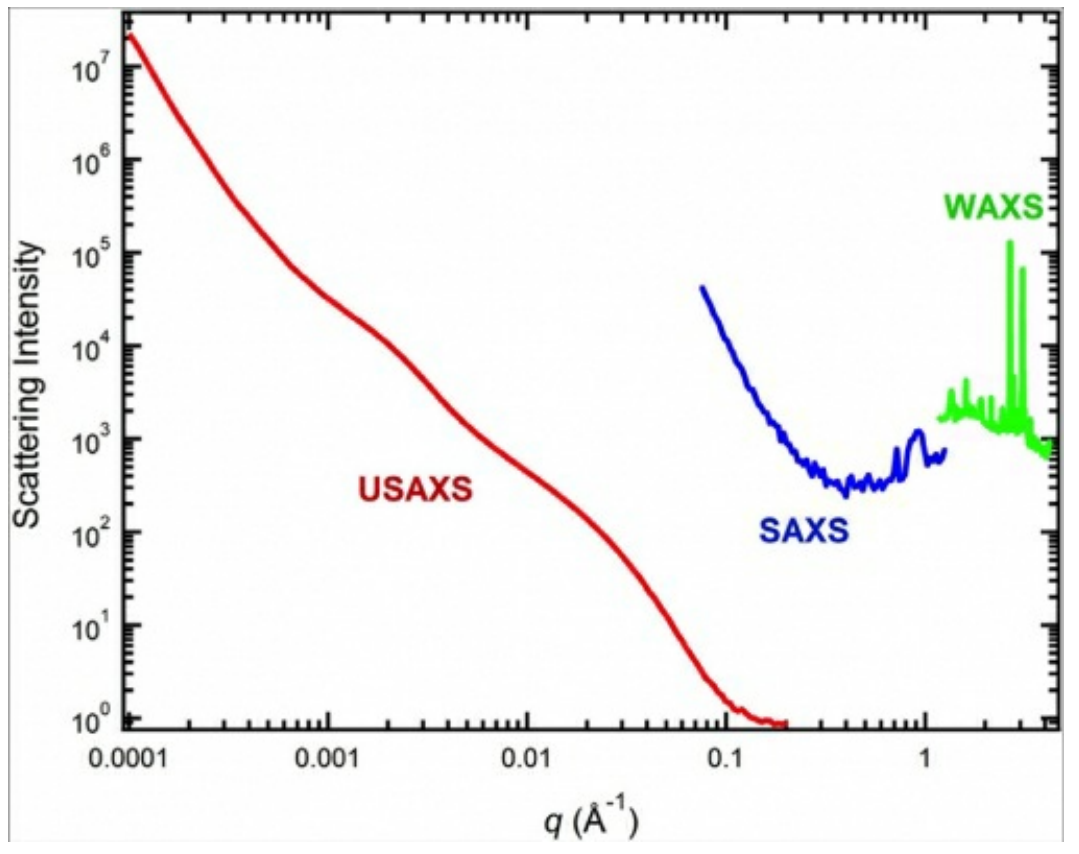

Keywords: advanced alloys, USAXS/SAXS/WAXS, extended range characterization 